Normal forms of lienard type for analytic unfoldings of nilpotent singularities Peer-reviewed author version

HUZAK, Renato (2017) Normal forms of lienard type for analytic unfoldings of nilpotent singularities. In: PROCEEDINGS OF THE AMERICAN MATHEMATICAL SOCIETY,145 (10), p. 4325-4336.

DOI: $10.1090 / \mathrm{proc} / 13539$

Handle: http://hdl.handle.net/1942/24039 


\title{
Normal forms of Liénard type for analytic unfoldings of nilpotent singularities
}

\author{
Renato Huzak
}

\begin{abstract}
Using the technique of gluing complex manifolds (equipped with vector fields) developed by Loray and the theory of deformation of complex structures developed by Kodaira and Spencer, we find normal forms of Liénard type for analytic unfoldings of planar singularities with a nonradial linear part. In particular, we improve normal forms of Takens for analytic unfoldings of nilpotent singularities and normal forms of De Maesschalck, Dumortier and Roussarie for analytic unfoldings of nilpotent contact points in planar slow-fast systems.
\end{abstract}

\section{Introduction}

Liénard equations appear in virtually every area of science. They are also a good starting point to try to solve the second part of Hilbert's 16th problem which is in essence to determine maximal number of limit cycles a planar polynomial vector field may have if the polynomial degree of the vector field is given (see [Sma00]). The principal goal of our paper is to give a positive answer to the following question that naturally arises in planar slow-fast setting: Is it true that all analytic slow-fast structures with nilpotent contact points can be understood by studying only (slow-fast) Liénard equations? We point out that much progress has been made in the study of slow-fast Liénard equations due to their simpler slow-fast sturucture (see e.g. [DR96], [KS01], [DR01], [DPR07], [DR09], [DMD11], [DMH14], [HDM14],...). Now, taking into account our result, the results on (slow-fast) Liénard equations become more relevant to understanding general nilpotent contact points.

In 2006, Loray published a paper in which he found a normal form of Liénard type for a planar analytic vector field near singularity at which its linear part is not radial, i.e. not of the form $\rho x \partial_{x}+\rho y \partial_{y}$, in the real and complex setting (see [Lor06], Theorem 4). Inspired by his result, we prove a similar result for analytic families of planar vector fields.

Theorem 1. Let $X_{\lambda}=f(x, y, \lambda) \partial_{x}+g(x, y, \lambda) \partial_{y}$ be an analytic $\lambda$-family of vector fields defined near the origin $(x, y, \lambda)=(0,0,0)$ of $\mathbb{R}^{2+p}$ (resp. of $\left.\mathbb{C}^{2+p}\right)$. If $f(0,0,0)=g(0,0,0)=0$ and the order of vanishing of $f(0, y, 0)$ at $y=0$ is one, then there exists a local analytic $\lambda$-family of coordinate changes of the form

$$
(x, y) \mapsto\left(\phi_{1}(x, y, \lambda), \phi_{2}(x, y, \lambda)\right)
$$


bringing $X_{\lambda}$ in an analytic $\lambda$-family of Liénard vector fields (up to multiplication by an analytic nowhere zero function)

$$
(y-F(x, \lambda)) \partial_{x}+G(x, \lambda) \partial_{y}
$$

where $F, G \in \mathbb{R}\{x, \lambda\}$ (resp. $F, G \in \mathbb{C}\{x, \lambda\})$ and $F(0,0)=G(0,0)=0$.

Remark 1. In fact, the condition on the order of vanishing of $f(0, y, 0)$ at $y=0$ in Theorem 1 is equivalent to nonradiality of the linearized vector field of $X_{0}$ at the origin. Indeed, given a nonradial linear part, there is a linear change of coordinates that converts the original family $X_{\lambda}$ into one with $\frac{\partial f}{\partial y}(0,0,0) \neq 0$. Conversely, $\frac{\partial f}{\partial y}(0,0,0) \neq 0$ implies nonradiality of the linear part.

Remark 2. If we introduce the new variable $Y=y-F(x, \lambda)$ and write $\widetilde{F}(x, \lambda)=$ $-\frac{\partial}{\partial x} F(x, \lambda)$, then the normal form (1) changes into the vector field

$$
Y \partial_{x}+(\widetilde{F}(x, \lambda) Y+G(x, \lambda)) \partial_{Y}
$$

which we will often use throughout the paper.

Theorem 1 will be proved in Section 2 .

The normal form (1) in Theorem 1 is valid in a fixed neighborhood of the origin in $\mathbb{R}^{2}$ or $\mathbb{C}^{2}$, independent of the parameter $\lambda$. If $f(x, y, \lambda)=f(x, y, 0)$, $g(x, y, \lambda)=g(x, y, 0)$ for all $\lambda \sim 0$, the normal form (1) can be found in [Lor06] (Theorem 4). To prove Theorem 1, we use the following method explained in [Lor06]. Given a holomorphic vector field $X_{\lambda}+0 \frac{\partial}{\partial \lambda}$ near the origin, we extend it, in the $y$-direction, to a meromorphic unfolding on a $(2+p)$-dimensional complex manifold $C$ which is a tubular neighborhood of an embedded Riemann sphere $\overline{\mathbb{C}}$. As we cannot construct a $\overline{\mathbb{C}}$-fibration at the same time, we are not allowed to use directly Grauert-Fischer Theorem (see [FG65]) to conclude the local triviality of the neighborhood of $\overline{\mathbb{C}}$. In [Lor06], the following theorem of V. I. Savel'ev (see [Sav82]) has been used to prove that the tubular neighborhood of an embedded Riemann sphere $\overline{\mathbb{C}}$ is biholomorphically equivalent to a direct product of the sphere $\overline{\mathbb{C}}$ and the disk: 2-dimensional complex manifold with an embedded Riemann sphere $\overline{\mathbb{C}}$ with trivial normal bundle in the complex manifold is a trivial $\overline{\mathbb{C}}$-bundle over the disc. As we deal with a higer dimensional ambient space $C$ surrounding an embedded sphere $\overline{\mathbb{C}}$, we have to use a result of Kodaira and Spencer (see $[$ Kod86] or [KS58]) which enables us to conclude a local triviality of the $(2+p)$-dimensional neighborhood of the embedded Riemann sphere $\overline{\mathbb{C}}$ from the triviality of the normal bundle of the embedded sphere. In fact, if we assume that the normal bundle of the embedded sphere $\overline{\mathbb{C}}$ in the complex manifold $C$ is trivial, then $C$ can be considered as a complex analytic fibre space, i.e., a complex analytic family, with one fiber being $\overline{\mathbb{C}}$. This is true because the cohomology group $H^{1}(\overline{\mathbb{C}}, \mathcal{O})=0$ where $\mathcal{O}$ is the sheaf of germs of holomorphic functions on $\overline{\mathbb{C}}$ (see e.g. [Gri65]). Hence we may use the theory of deformation of complex structures [Kod86] developed by Kodaira and Spencer. It is well known (see $[\operatorname{Kod} 86]$, Chapter 4 ) that the complex analytic family $C$ is trivial if it is trivial as a differentiable family. Since $H^{1}(\overline{\mathbb{C}}, \Theta)=0$ where $\Theta$ is the sheaf of germs of holomorphic vector fields on $\overline{\mathbb{C}}$ (see [Bot57]), the Rigidity Theorem of Frölicher-Nijenhuis (see $[\operatorname{Kod} 86]$, Theorem 4.5.) implies triviality of the differentiable family $C$, i.e., triviality of complex analytic family $C$. The 
theory of Kodaira and Spencer also implies that the global coordinates send $C$ to a neighborhood of $\{0\} \times \overline{\mathbb{C}} \times\{0\}$ in the Cartesian product $\mathbb{C} \times \overline{\mathbb{C}} \times \mathbb{C}^{p}$ preserving $\lambda$ (see Section 2). Thus the original meromorphic unfolding can be transformed in a new meromorphic unfolding. The new meromorphic unfolding is rational in $y$ and the rest of the proof of Theorem 1 is very similar to the proof of Theorem 4 in [Lor06].

Grauert-Fischer Theorem (hence without referring to the result of KodairaSpencer) allows us to prove a weaker version of Theorem 1 in which multiplication of vector field by a meromorphic function is allowed.

Theorem 2. Let $X_{\lambda}=f(x, y, \lambda) \partial_{x}+g(x, y, \lambda) \partial_{y}$ be an analytic $\lambda$-family of vector fields defined near the origin $(x, y, \lambda)=(0,0,0)$ of $\mathbb{R}^{2+p}$ (resp. of $\mathbb{C}^{2+p}$ ). Under the assumptions of Theorem 1, there exists a local analytic $\lambda$-family of coordinate changes bringing $X_{\lambda}$ in the form (1) where $F, G \in \mathbb{R}\{x, \lambda\}$ (resp. $F, G \in \mathbb{C}\{x, \lambda\}$ ) and $F(0,0)=G(0,0)=0$ (up to multiplication by a meromorphic function).

Theorem 2 will be proved in Section 3 .

Let us focus on a real analytic $\lambda$-family of planar vector fields $X_{\lambda}$ near the origin of $\mathbb{R}^{2}$, with $\lambda \in \mathbb{R}^{p}$ and $\lambda \sim 0$. We suppose that $X_{0}$ has a nilpotent singularity at the origin, i.e. both eigenvalues of $D X_{0}(0,0)$ are equal to 0 and $D X_{0}(0,0) \not \equiv 0$. Using the Jordan normal form theorem we may assume that the linearized vector field of $X_{0}$ at the origin has the canonical form $y \partial_{x}$ :

$$
X_{\lambda}=(y+p(x, y, \lambda)) \partial_{x}+q(x, y, \lambda) \partial_{y}
$$

where $p$ and $q$ are analytic and $p(x, y, 0)$ and $q(x, y, 0)$ are $O\left(\|(x, y)\|^{2}\right)$. There exist analytic coordinates $(X, Y)=(x, y+p(x, y, \lambda))$ in which

$$
X_{\lambda}=y \partial_{x}+\left(q_{1}(x, \lambda)+y q_{2}(x, \lambda)+y^{2} q_{3}(x, y, \lambda)\right) \partial_{y}
$$

where $(X, Y)$ is denoted by $(x, y), q_{1}, q_{2}$ and $q_{3}$ are analytic functions, $q_{1}(x, 0)=$ $O\left(x^{2}\right)$ and $q_{2}(x, 0)=O(x)$. A well known result is the following theorem of Takens (see [Tak74] and [Dum]): Consider an analytic unfolding of the nilpotent singularity, i.e., a family $X_{\lambda}$ with $X_{0}$ having a nilpotent singularity at the origin of $\mathbb{R}^{2}$. For any $n$ large enough $X_{\lambda}$ is analytically conjugate (respecting the parameter $\lambda$ ) to

$$
y \partial_{x}+\left(F(x, \lambda)+y G(x, \lambda)+y^{2} Q(x, y, \lambda)\right) \partial_{y},
$$

where $F, G$ and $Q$ are analytic functions, $F(x, 0)=O\left(x^{2}\right), G(x, 0)=O(x)$ and $Q=O\left((\|(x, y)\|+\|\lambda\|)^{n}\right)$.

Theorem 1 and Remark 2 imply

Theorem 3. There is an analytic $\lambda$-family of coordinate changes bringing (2), up to multiplication by an analytic nowhere zero function, in the form

$$
y \partial_{x}+\left(q_{1}(x, \lambda)+y q_{2}(x, \lambda)\right) \partial_{y}
$$

where $q_{1}$ and $q_{2}$ are new analytic functions, $q_{1}(x, 0)=O\left(x^{2}\right)$ and $q_{2}(x, 0)=$ $O(x)$. 
Thus, at the level of analytic equivalences, the normal form (4) of Liénard type simplifies Takens normal form (3).

Remark 3. When $X_{\lambda}$ has a nilpotent singularity at the origin for each $\lambda \sim 0$, then, for any fixed $\lambda$, there exists an analytic change of coordinates bringing $X_{\lambda}$

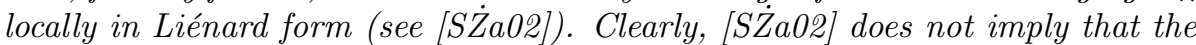
$\lambda$-family of analytic changes of coordinates is valid in a $\lambda$-uniform neighborhood of the origin in the phase space.

In planar slow-fast systems $X_{\epsilon, \mu}(\lambda=(\epsilon, \mu))$ a curve of singularities, called the critical curve, appears for $\epsilon=0$ where $\epsilon$ is a singular perturbation parameter. The critical curve typically consists of normally hyperbolic singularities (the linearized vector field at a normally hyperbolic singularity has one zero eigenvalue with corresponding eigenvector tangent to the critical curve) and one contact point (often called turning point). The linearized vector field at the contact point has two zero eigenvalues and we suppose it is of nilpotent type for $\mu=0$. It is shown in [DMDR11] that any analytic family of planar slow-fast vector fields $X_{\epsilon, \mu}$, locally near the nilpotent contact point for $(\epsilon, \mu) \sim(0,0)$, is analytically equivalent (preserving $(\epsilon, \mu))$ to the following normal form:

$$
(y-f(x, \mu)) \partial_{x}+\epsilon(g(x, \epsilon, \mu)+(y-f(x, \mu)) h(x, y, \epsilon, \mu)) \partial_{y},
$$

for analytic functions $f, g$ and $h$ and $f(0,0)=\partial_{x} f(0,0)=0$. If the $\partial_{y^{-}}$ component of (5) does not depend on $y$, we deal with generalized slow-fast Liénard vector fields. A motivation to study the slow-fast Liénard vector fields can be found in [Dum06] and [Rou07].

Our goal is to find a normal form of Liénard type for (5) in a neighborhood of the origin in the $(x, y)$-plane that does not shrink to the origin when $\epsilon \rightarrow 0$ and $\mu \rightarrow 0$. We call the order of vanishing of $f(x, 0)$ at $x=0$, which is $\geq 2$, order of the contact point and the order of vanishing of $g(x, 0,0)$ at $x=0$, which is $\geq 0$, the singularity order at the contact point (see [DMDR11]). If a nilpotent contact point of (5) is of order two (or of Morse-type), the analytic vector field (5) can locally near the origin be written as a slow-fast Liénard vector field up to an $\epsilon$-exponentially small remainder (see [DM14], Theorem 2). Furthermore, if the singularity order at the contact point is zero (i.e. the contact point is a slow-fast jump point), then the exponentially small remainder can be eliminated (see [DM14], Theorem 3). In [DMD16], the results obtained in [DM14] have been generalized to nilpotent contact points of an arbitrary order $n$, to so-called non-generic nilpotent contact points. More precisely, it is possible to bring (5), locally near the non-generic contact point, in Liénard form up to exponentially small error. For non-generic jump points $(g(0,0,0) \neq 0)$, a normal form of Liénard type has been obtained. The results in [DM14] and [DMD16] have been proven by using Gevrey asymptotics. Without computation, we eliminate the exponentially small remainder in the normal forms obtained in [DM14] and [DMD16]. Moreover, we improve smoothness of local coordinate changes and regular changes of time needed in [DM14] and [DMD16] to bring (5) in the Liénard form in the jump case.

Theorem 4. There exists a local analytic $(\epsilon, \mu)$-family of coordinate changes bringing (5), up to multiplication by an analytic nowhere zero function, in the Liénard form

$$
(y-\widetilde{f}(x, \epsilon, \mu)) \partial_{x}+\epsilon \widetilde{g}(x, \epsilon, \mu) \partial_{y}
$$


for some analytic functions $\tilde{f}$ and $\widetilde{g}$ such that the order of $\tilde{f}(x, 0,0)$ at $x=0$ (resp. of $\widetilde{g}(x, 0,0)$ at $x=0$ ) is equal to the order of $f(x, 0)$ at $x=0$ (resp. of $g(x, 0,0)$ at $x=0)$.

Theorem 4 will be proved in Section 4 .

\section{Proof of Theorem 1}

Let $X_{\lambda}=f(x, y, \lambda) \partial_{x}+g(x, y, \lambda) \partial_{y}$ be an analytic $\lambda$-family of vector fileds where $(x, y, \lambda) \sim(0,0,0) \in \mathbb{C}^{2+p}$ and $f(0, y, 0)$ vanishes at the order 1 at 0 . Since $\frac{\partial f}{\partial y}(0,0,0) \neq 0$, the Implicit Function Theorem implies existence of an analytic function $y=\phi(Y, \lambda), \phi(Y, 0)$ vanishing at the order 1 at 0 , such that $f(0, \phi(Y, \lambda), \lambda)=Y$. Therefore, after a change of the $y$-coordinate of the form $y=\phi(Y, \lambda)$, we may assume that $f(0, y, \lambda)=y$ in $X_{\lambda}$.

Following [Lor06], we define the line $l=\{0\} \times \overline{\mathbb{C}} \times\{0\}$ in $\mathbb{C} \times \overline{\mathbb{C}} \times \mathbb{C}^{p}$ and the covering $l_{0}=\{0\} \times\{|y|<r\} \times\{0\}$ and $l_{\infty}=\{0\} \times\left\{|y|>\frac{r}{2}\right\} \times$ $\{0\}$ where $r>0$. The family $X_{\lambda}$ is well-defined on a neighborhood of the closure of $l_{0}$ by taking $r$ small enough. Rectification Theorem (or CauchyKovalevskaya Theorem) implies existence of a unique diffeomorphism defined on a neighborhood of $l_{0} \cap l_{\infty}$ in $\mathbb{C} \times \overline{\mathbb{C}} \times \mathbb{C}^{p}$ and of the form

$$
(\bar{x}, \bar{y}, \lambda)=\Psi(x, y, \lambda)=\left(\psi_{1}(x, y, \lambda), \psi_{2}(x, y, \lambda), \lambda\right), \Psi(0, y, \lambda)=(0, y, \lambda)
$$

conjugating $X_{\lambda}+0 \partial_{\lambda}$ to the vector field $X^{\infty}=\bar{y} \partial_{\bar{x}}+0 \partial_{\bar{y}}+0 \partial_{\lambda}$. Indeed, $f(0, y, \lambda) \neq 0$ for $y \neq 0$.

The following theorem (see [FG02], Chapter IV) enables us to glue the Cartesian product $\mathbb{C} \times \overline{\mathbb{C}} \times \mathbb{C}^{p}$ near $l_{0}$ (denoted by $M_{1}$ ) and the Cartesian product $\mathbb{C} \times \overline{\mathbb{C}} \times \mathbb{C}^{p}$ near $l_{\infty}\left(\right.$ denoted by $\left.M_{2}\right)$ along $l_{0} \cap l_{\infty}$ by means of $\Psi$ and obtain a global complex manifold $C$ of dimension $2+p$ along $l$.

Theorem 5. Let $C$ be a set that is the union of a countable collection $\left(C_{\rho}\right)_{\rho \in \mathbb{N}}$ of subsets such that:

1. For every $\rho \in \mathbb{N}$, there exists a bijection $f_{\rho}: C_{\rho} \rightarrow M_{\rho}$, for an $n$ dimensional complex manifold $M_{\rho}$;

2. For every $\left(\rho_{1}, \rho_{2}\right) \in \mathbb{N} \times \mathbb{N}$ the subset $f_{\rho_{1}}\left(C_{\rho_{1}} \cap C_{\rho_{2}}\right)$ is open in $M_{\rho_{1}}$, and the map

$$
f_{\rho_{1}} \circ f_{\rho_{2}}^{-1}: f_{\rho_{2}}\left(C_{\rho_{1}} \cap C_{\rho_{2}}\right) \rightarrow f_{\rho_{1}}\left(C_{\rho_{1}} \cap C_{\rho_{2}}\right)
$$

is biholomorphic;

3. For all $a \in C_{\rho_{1}}$ and $b \in C_{\rho_{2}}, a \neq b$, there are open neighborhoods $f_{\rho_{1}}(a) \in$ $U \subset M_{\rho_{1}}$ and $f_{\rho_{2}}(b) \in V \subset M_{\rho_{2}}$ with $f_{\rho_{1}}^{-1}(U) \cap f_{\rho_{2}}^{-1}(V)=\varnothing$.

Then there exists a unique complex structure on $C$ such that the subsets $C_{\rho}$ are open in $C$ and the maps $f_{\rho}: C_{\rho} \rightarrow M_{\rho}$ are biholomorphic. (One says that $C$ is obtained by gluing the complex manifolds $M_{\rho}$.)

Indeed, we define a set $C:=C_{1} \cup C_{2}$ with

$$
C_{1}=\{(x,(1: y), \lambda)|x \sim 0,| y \mid<r, \lambda \sim 0\}
$$


and

$$
C_{2}=\left\{(x,(1: y), \lambda)|x \sim 0,| y \mid>\frac{r}{2}, \lambda \sim 0\right\} \cup\{(x,(0: 1), \lambda) \mid x \sim 0, \lambda \sim 0\},
$$

where $(1: y), y \in \mathbb{C}$, and $(0: 1)=\infty$ are elements of the set $\overline{\mathbb{C}}$. The bijection $f_{1}: C_{1} \rightarrow M_{1}$ is given by the identity function on $C_{1}$. On $C_{1} \cap C_{2}$, the bijection $f_{2}: C_{2} \rightarrow M_{2}$ is given by

$$
f_{2}(x,(1: y), \lambda)=\left(\psi_{1}(x, y, \lambda),\left(1: \psi_{2}(x, y, \lambda)\right), \lambda\right) .
$$

Now, it can be easily seen that all three conditions in Theorem 5 are satisfied. Hence, there exists a complex structure on $C$ with the properties given in Theorem 5 .

The tangent bundle $T(C)$ of the complex manifold $C$ is given by the transition function $J_{\widetilde{\Psi}}$ with respect to the open covering $\left\{C_{1}, C_{2}\right\}$ of $C$ where

$$
\widetilde{\Psi}(x, y, \lambda):=\left(\psi_{1}(x, y, \lambda), \frac{1}{\psi_{2}(x, y, \lambda)}, \lambda\right) .
$$

Furthermore, the holomorphic vector field $X_{\lambda}+0 \partial_{\lambda}$ on $M_{1}$ (resp. the meromorphic vector field $X^{\infty}$ on $M_{2}$ ) induces a holomorphic section $s_{1}$ in $T(C)$ over $C_{1}$ (resp. a meromorphic section $s_{2}$ in $T(C)$ over $C_{2}$ ) such that

$$
s_{1}(x,(1: y), \lambda)=(f(x, y, \lambda), g(x, y, \lambda), 0)
$$

on $C_{1}$ (resp.

$$
s_{2}(x,(1: y), \lambda)=\left(\psi_{2}(x, y, \lambda), 0,0\right)
$$

on $C_{1} \cap C_{2}$ ). On $C_{1} \cap C_{2}$, we have the following compatibility condition

$$
s_{2}(x,(1: y), \lambda)=s_{1}(x,(1: y), \lambda)\left(J_{\widetilde{\Psi}}(x, y, \lambda)\right)^{t} .
$$

Hence, the system $\left(s_{1}, s_{2}\right)$ defines a (global) meromorphic section in $T(C)$ over $C$, i.e., a meromorphic vector field $X$ on $C$.

Since $f(0, y, \lambda)=y$ and $\Psi(0, y, \lambda)=(0, y, \lambda)$, the transition function $J_{\widetilde{\Psi}}$ for the tangent bundle $T(C)$ has the following form, along $l_{0} \cap l_{\infty}$ :

$$
J_{\widetilde{\Psi}}(0, y, 0)=\left(\begin{array}{ccc}
1 & 0 & 0 \\
* & -\frac{1}{y^{2}} & 0 \\
0 & 0 & I_{p}
\end{array}\right)
$$

where $I_{p}$ is a $p \times p$ identity matrix. Therefore, the transition function for the normal bundle of the embedded sphere $l$ in $C$ is given by $\frac{\partial\left(\psi_{1}, \lambda\right)}{\partial(x, \lambda)}(0, y, 0)=I_{p+1}$ where $I_{p+1}$ is a $(p+1) \times(p+1)$ identity matrix (see also [FG02], Chapter IV), i.e., the normal bundle has to be trivial. As explained in Introduction, there exist global coordinates $(x, y, \lambda)$ sending $C$ in a neighborhood of the embedded sphere $l$ onto the Cartesian product $\mathbb{C} \times \overline{\mathbb{C}} \times \mathbb{C}^{p}$ near $\{0\} \times \overline{\mathbb{C}} \times\{0\}$, sending $l$ onto $\{x=0, \lambda=0\}$ and preserving $\lambda$.

Remark 4. Let's explain why the global coordinates preserve $\lambda$. We focus on the proof of the Rigidity Theorem of Frölicher-Nijenhuis given in [Kod86]. 
More precisely, the Rigidity Theorem of Frölicher-Nijenhuis follows from Theorem 4.6. in [Kod86] if $H^{1}\left(C_{0}, \Theta_{0}\right)=0$ : "Theorem 4.6. If $\operatorname{dim} H^{1}\left(C_{t}, \Theta_{t}\right)$ is independent of $t \in B \subset \mathbb{C}^{m}$, and $\rho_{t}=0$ identically, then the complex analytic family $(C, B, \omega)$ is locally trivial". $\rho_{t}$ is the "derivative" of the complex structure $C_{t}$ with respect to $t$ (we refer to [Kod86] for a precise definition of $\rho_{t}$ ). In our case, $C$ is the complex manifold od dimension $(2+p)$ defined in this section, $B$ is a domain in $\mathbb{C}^{p+1}, 0 \in B$, and $t=\left(t_{1}, \ldots, t_{p+1}\right)=\omega(P), P \in C$, is a holomorphic map of $C$ onto $B$ such that $C_{t}=\omega^{-1}(t)$ is a compact complex submanifold of $C$ and the rank of the Jacobian of $\omega$, in terms of local complex coordinates $\left(z_{1}(P), \ldots, z_{p+2}(P)\right)$ of $C$, is equal to $p+1$ at every point of $C$. Thus after an appropriate renumbering of $z_{i}$ 's, we can choose the following system of local complex coordinates of $C$ :

$$
\left(z_{1}(P), t_{1}, \ldots, t_{p+1}\right), \omega(P)=\left(t_{1}, \ldots, t_{p+1}\right) .
$$

See [Kod86]. In terms of these coordinates, $\omega$ is the projection

$$
\omega\left(z_{1}, t_{1}, \ldots, t_{p+1}\right)=\left(t_{1}, \ldots, t_{p+1}\right)
$$

and $\left(t_{1}, \ldots, t_{p+1}\right)$ as part of local coordinates on the complex manifold $C$ do not change under coordinate transformations. In the proof of Theorem 4.6., a biholomorphic map $\Phi: \overline{\mathbb{C}} \times \Delta \rightarrow \omega^{-1}(\Delta)$ is defined by virtue of the solution of ordinary differential equations, where $\Delta$ is a small polydisk with $0 \in \Delta \subset B$ and where $\Phi$ maps $\overline{\mathbb{C}} \times t$ onto $C_{t}=\omega^{-1}(t)$. Thus the global coordinates $\Phi$ preserve $t=\left(t_{1}, \ldots, t_{p+1}\right)$. Now it suffices to prove that we can take $\left(t_{2}, \ldots, t_{p+1}\right)=\lambda$. Indeed, since $\lambda$ is invariant under $\Psi$, the local coordinates of $C$ given by the maps $f_{1}$ and $f_{2}$, defined in this section, preserve $\lambda$. It is clear now that we can take $\omega(x, y, \lambda)=(*, \lambda)$. Note that the rank of $\omega$ is equal to $p+1$ because the normal bundle is trivial.

In other words, the complex manifold $C$ is biholomorphically equivalent to a trivial $\overline{\mathbb{C}}$-bundle over a polydisk with center $0 \in \mathbb{C}^{p+1}$. It implies that the coefficients of the meromorphic vector field $X=P_{1}(x, y, \lambda) \partial_{x}+P_{2}(x, y, \lambda) \partial_{y}+$ $0 \partial_{\lambda}$ have to be rational in $y$ (see [FG02]). Without loss of generality we may assume that the unique and simple pole of $X$ (given by the meromorphic vector field $X^{\infty}$ ) is given by $\{y=\infty\}$ (we can use the coordinate change $Y=\frac{a y+b}{c y+d}$ where $a, b, c, d \in \mathbb{C}\{x, \lambda\}$ and $a d-b c \not \equiv 0)$. Then we claim $P_{1}$ (resp. $P_{2}$ ) is a polynomial in $y$ of maximal degree 1 (resp. 3). Indeed, the change of coordinates $Y=\frac{1}{y}$ changes $X$ to

$$
P_{1}\left(x, \frac{1}{Y}, \lambda\right) \partial_{x}-Y^{2} P_{2}\left(x, \frac{1}{Y}, \lambda\right) \partial_{Y}+0 \partial_{\lambda}
$$

Hence, near the simple pole $\{y=\infty\}=\{Y=0\}$ of $X$, we have the following convergent Laurent expansions of $P_{1}$ and $P_{2}$ :

$$
\begin{gathered}
P_{1}\left(x, \frac{1}{Y}, \lambda\right)=a_{-1} \frac{1}{Y}+a_{0}+\cdots \\
P_{2}\left(x, \frac{1}{Y}, \lambda\right)=b_{-3} \frac{1}{Y^{3}}+b_{-2} \frac{1}{Y^{2}}+b_{-1} \frac{1}{Y}+b_{0}+\cdots
\end{gathered}
$$

where $a_{i}, b_{j} \in \mathbb{C}\{x, \lambda\}$. Since functions $P_{1}(x, y, \lambda)-a_{-1} y$ and $P_{2}(x, y, \lambda)-$ $b_{-3} y^{3}-b_{-2} y^{2}-b_{-1} y$ have no poles, they are holomorphic on (compact and 
connected) $\overline{\mathbb{C}}$, for each fixed $x \sim 0$ and $\lambda \sim 0$, that is, they are constants in $y$ (see [FG02]). Thus we have obtained

$$
X=\left(a_{0}+a_{1} y\right) \partial_{x}+\left(b_{0}+b_{1} y+b_{2} y^{2}+b_{3} y^{3}\right) \partial_{y}+0 \partial_{\lambda} .
$$

for new $a_{i}, b_{j} \in \mathbb{C}\{x, \lambda\}$.

A translation of the global coordinates allows us to assume that the coordinate change from the original coordinates near the origin in $\mathbb{C}^{2+p}$ to the global coordinates sends the origin in $\mathbb{C}^{2+p}$ to the origin in $\mathbb{C} \times \overline{\mathbb{C}} \times \mathbb{C}^{p}$. Hence, the vector field $X$ has a singularity at the origin $(x, y, \lambda)=(0,0,0)$ (note that $f(0,0,0)=g(0,0,0)=0)$. It is clear that the coordinate change preserves the $y$-axis near the singularity. From this together with $\frac{\partial f}{\partial y}(0,0,0) \neq 0$ we conclude that $a_{1}(0,0) \neq 0$ and, after the coordinate change $Y=y+\frac{a_{0}}{a_{1}}$, we may assume that $a_{0} \equiv 0$ in the expression for $X$.

Using the coordinate change $y=\frac{a Y}{Y+1}$ (with $a$ to be determined), and a time rescaling (multiplication by $(Y+1)$ ), we arrive at the following vector field

$$
\widetilde{a}_{1} Y \partial_{x}+\left(\widetilde{b}_{0}+\widetilde{b}_{1} Y+\widetilde{b}_{2} Y^{2}+\widetilde{b}_{3} Y^{3}\right) \partial_{Y}+0 \partial_{\lambda}
$$

where $\widetilde{a}_{1}, \widetilde{b}_{j} \in \mathbb{C}\{x, \lambda\}$, and where

$$
\widetilde{b}_{3}=a^{2} b_{3}+a b_{2}+b_{1}+\frac{b_{0}}{a}-a_{1} \frac{\partial}{\partial x} a .
$$

Now, Cauchy-Kovalevskaya Theorem implies existence of a function $a \in \mathbb{C}\{x, \lambda\}$ such that $a(0,0) \neq 0$ and $\widetilde{b}_{3} \equiv 0$. Indeed, we have $a_{1}(0,0) \neq 0$. To remove the quadratic term in the $\partial_{Y}$-component of $(6)$, we use the coordinate change $\widetilde{Y}=\exp \left(-\int_{0}^{x} \frac{\widetilde{b}_{2}(s, \lambda)}{\widetilde{a}_{1}(s, \lambda)} d s\right) Y$. Hence, the vector field (6) changes into

$$
\widetilde{a}_{1} \widetilde{Y} \partial_{x}+\left(\widetilde{b}_{0}+\widetilde{b}_{1} \tilde{Y}\right) \partial_{\tilde{Y}}+0 \partial_{\lambda}
$$

for new $\widetilde{a}_{1}, \widetilde{b}_{j} \in \mathbb{C}\{x, \lambda\}$ and $\widetilde{a}_{1}(0,0) \neq 0$. After a change of the $x$-coordinate, we may also assume that $\widetilde{a}_{1} \equiv 1$. Indeed, we use the coordinate change $x=\phi(\widetilde{x}, \lambda)$ where $\frac{\partial}{\partial \widetilde{x}} \phi(\widetilde{x}, \lambda)=\widetilde{a}_{1}(\phi(\widetilde{x}, \lambda), \lambda)$ and $\phi(0, \lambda)=0$. This proves the result in complex setting (see Remark 2).

When $X_{\lambda}=f(x, y, \lambda) \partial_{x}+g(x, y, \lambda) \partial_{y}$ is a real analytic family of planar vector fields, then $\overline{f(\bar{x}, \bar{y}, \bar{\lambda})}=f(x, y, \lambda)$ and $\overline{g(\bar{x}, \bar{y}, \bar{\lambda})}=g(x, y, \lambda)$. The same is true for the vector field $X^{\infty}=y \partial_{x}$. Hence $\overline{\Psi(\bar{x}, \bar{y}, \bar{\lambda})}=\Psi(x, y, \lambda)$, i.e., the gluing map $\Psi$ commutes with the antiholomorphic involution $(x, y, \lambda) \rightarrow(\bar{x}, \bar{y}, \bar{\lambda})$, due to uniqueness of $\Psi$. Then $\Psi$ induces an antiholomorphic involution $\eta: C \rightarrow C$ on the complex manifold $C$ such that $\bar{X}=\eta^{*} X$. Indeed, we define $\eta(p)=$ $c_{i}^{-1}\left(\overline{c_{i}(p)}\right), i=1,2$, where $c_{i}$ are local coordinates of $p \in C$. It is clear from the property of $\Psi$ that $\eta$ is an antiholomorphic involution, independent of the choice of local coordinates, satisfying $\bar{X}=\eta^{*} X$.

Since the coordinate $\lambda$ is invariant under $\Psi$, we have

$$
\eta(x, y, \lambda)=\left(\eta_{1}(x, y, \lambda), \eta_{2}(x, y, \lambda), \bar{\lambda}\right)
$$

By Blanchard's argument (see [Lor06]), the antiholomorphic involution $\eta$ sends a fiber to a fiber. Thus the first component $\eta_{1}$ of $\eta$ has the form $\eta_{1}(x, \lambda)$. 
For each fixed $\lambda$, the antiholomorphic function $\eta_{1}(x, \lambda)$ is a symmetry with respect to a real analytic curve, and, after a holomorphic change of the $x$ coordinate, $\eta_{1}(x, \lambda)=\bar{x}$. On the other hand, for each fixed $(x, \lambda), \eta_{2}(x, y, \lambda)$ is a reflection with respect to a Möbius circle, and, after a holomorphic change of the $y$-coordinate, we have $\eta_{2}(x, y, \lambda)=\bar{y}$. Hence $\eta(x, y, \lambda)=(\bar{x}, \bar{y}, \bar{\lambda})$. Since $\bar{X}=\eta^{*} X$, the meromorphic vector field $X$ has real coefficients. See also [Lor06], proof of Theorem 4 .

\section{Proof of Theorem 2}

Let $X_{\lambda}=f(x, y, \lambda) \partial_{x}+g(x, y, \lambda) \partial_{y}$ be an analytic $\lambda$-family of vector fileds at $(x, y, \lambda)=(0,0,0) \in \mathbb{C}^{2+p}$, vanishing at $(0,0,0)$, with $f(0, y, 0)$ vanishing at the order 1 at 0 . After a change of the $y$-coordinate of the form $y=\phi(x, Y, \lambda)$, we may take $f(x, y, \lambda) \equiv y$. Indeed, the Implicit Function Theorem implies existence of a unique analytic function $y=\phi(x, Y, \lambda)$ such that $\phi(0,0,0)=0$, $\frac{\partial \phi}{\partial Y}(0,0,0) \neq 0$ and $f(x, \phi(x, Y, \lambda), \lambda)=Y$ (note that $\left.\frac{\partial f}{\partial y}(0,0,0) \neq 0\right)$.

As in the proof of Theorem 1, we define the line $l$ and the covering $\left\{l_{0}, l_{\infty}\right\}$ of $l$ such that $X_{\lambda}$ is well-defined on a neighborhood of the closure of $l_{0}$. As a simple consequence of the Flow-Box Theorem (or Cauchy-Kovalevskaya Theorem), we can find a unique diffeomorphism defined on a neighborhood of $l_{0} \cap l_{\infty}$, of the form

$$
\Psi(x, y, \lambda)=\left(x, \psi_{2}(x, y, \lambda), \lambda\right), \quad \Psi(0, y, \lambda)=(0, y, \lambda)
$$

conjugating the vector field

$$
\frac{1}{y} X_{\lambda}+0 \partial_{\lambda}=1 \partial_{x}+\frac{g(x, y, \lambda)}{y} \partial_{y}+0 \partial_{\lambda}
$$

to the vector field $X^{\infty}=1 \partial_{x}+0 \partial_{y}+0 \partial_{\lambda}$. Now we glue $\mathbb{C} \times \overline{\mathbb{C}} \times \mathbb{C}^{p}$ near $l_{0}$ and $\mathbb{C} \times \overline{\mathbb{C}} \times \mathbb{C}^{p}$ near $l_{\infty}$ along $l_{0} \cap l_{\infty}$ by means of $\Psi$ and obtain a global complex manifold $C$ of dimension $2+p$ along $l$ equipped with a meromorphic vector field $X$. Indeed, the argument used in the proof of Theorem 1 goes over essentially unchanged.

Since the coordinates $(x, \lambda)$ are invariant under $\Psi$, it follows that $(x, \lambda)$ define a rational fibration $(x, \lambda): C \rightarrow P^{p+1}$ where $P^{p+1}$ is a polydisk with center $0 \in \mathbb{C}^{p+1}$. Now Grauert-Fischer Theorem implies existence of a submersion $y: C \rightarrow l \simeq \overline{\mathbb{C}}$ that completes $(x, \lambda)$ into a system of trivializing coordinates $(x, \lambda, y): C \rightarrow P^{p+1} \times \overline{\mathbb{C}}$. See also [Lor06].

Hence, as in the proof of Theorem 1, the coefficients of the meromorphic vector field $X=P_{1}(x, y, \lambda) \partial_{x}+P_{2}(x, y, \lambda) \partial_{y}+0 \partial_{\lambda}$ have to be rational in the $y$-variable. Moreover, we may assume that a (possible) simple pole of $X$ (given by $\left.\frac{1}{y} X_{\lambda}+0 \partial_{\lambda}\right)$ is given by $\{y=0\}$ and that the coordinate change sends the origin in $\mathbb{C}^{2+p}$, near which the vector field $\frac{1}{y} X_{\lambda}+0 \partial_{\lambda}$ is defined, to the origin in $\mathbb{C} \times \overline{\mathbb{C}} \times \mathbb{C}^{p}$. Indeed, we use a change of the $y$-coordinate of the form $Y=\frac{a y+b}{c y+d}$ where $a, b, c, d \in \mathbb{C}\{x, \lambda\}$ and $a d-b c \not \equiv 0$. To find the form of rational functions $P_{1}$ and $P_{2}$ in the expression for $X$, we use the same trick as in the proof of Theorem 1. We subtract the principal part of a convergent Laurent expansion of $P_{1}$ near the simple pole $\{y=0\}$. What is left is a holomorphic function on $\overline{\mathbb{C}}$, for each $(x, \lambda)$. That has to be constant in the $y$-variable. Besides a simple pole at $\{y=0\}, P_{2}$ may have a pole at $\{y=\infty\}$ of maximal order 2. After 
subtracting the principal part of Laurent expansions of $P_{2}$ near $\{y=0\}$ and $\{y=\infty\}$, we are left with constant in $y$. Thus the vector field $X$ can be written as

$$
X=\frac{Q_{1}(x, y, \lambda)}{y} \partial_{x}+\frac{Q_{2}(x, y, \lambda)}{y} \partial_{y}+0 \partial_{\lambda}
$$

where $Q_{1}$ (resp. $Q_{2}$ ) is a polynomial in the $y$-variable of maximal degree 1 (resp. $3), Q_{1}(0,0,0)=Q_{2}(0,0,0)=0$. Now, if we multiply the vector field $X$ by $y$, we arrive at a polynomial vector field that can be brought in the Liénard form (1) (see the proof of Theorem 1).

Now suppose that $X_{\lambda}$ is a real analytic family of vector fields. Since the vector field $X^{\infty}=1 \partial_{x}+0 \partial_{y}+0 \partial_{\lambda}$ is also real, the gluing map $\Psi$, commuting with $(x, y, \lambda) \mapsto(\bar{x}, \bar{y}, \bar{\lambda})$, induces an antiholomorphic involution $\eta: C \rightarrow C$ on the complex manifold $C$ such that $\bar{X}=\eta^{*} X$. Note that the coordinates $(x, \lambda)$ are invariant under $\Psi$. We therefore have $\eta(x, y, \lambda)=\left(\bar{x}, \eta_{1}(x, y, \lambda), \bar{\lambda}\right)$ with an antiholomorphic function $\eta_{1}$. For each fixed $(x, \lambda), \eta_{1}$ is a reflection with respect to a Möbius circle, and we may assume that $\eta_{1}(x, y, \lambda)=\bar{y}$ after a holomorphic change of the $y$-coordinate. Thus we have $\eta(x, y, \lambda)=(\bar{x}, \bar{y}, \bar{\lambda})$, and, as a simple consequence of $\bar{X}=\eta^{*} X$, coefficients of the vector field $X$ have to be real. See also [Lor06], proof of Theorem 1.

\section{Proof of Theorem 4}

Denote the slow-fast vector field (5) by $X_{\epsilon, \mu}$. Following Theorem 1, there exists an analytic $(\epsilon, \mu)$-family of coordinate changes $(\widetilde{x}, \widetilde{y})=\psi^{\epsilon, \mu}(x, y)=$ $\left(\psi_{1}^{\epsilon, \mu}(x, y), \psi_{2}^{\epsilon, \mu}(x, y)\right)$ with $\psi^{0,0}(0,0)=(0,0)$, and a positive analytic function $\rho(\widetilde{x}, \widetilde{y}, \epsilon, \mu)$ such that

$$
\rho(\widetilde{x}, \widetilde{y}, \epsilon, \mu) . J_{\psi^{\epsilon, \mu}}\left(\left(\psi^{\epsilon, \mu}\right)^{-1}(\widetilde{x}, \widetilde{y})\right) X_{\epsilon, \mu}\left(\left(\psi^{\epsilon, \mu}\right)^{-1}(\widetilde{x}, \widetilde{y})\right)=\left(\begin{array}{c}
\widetilde{y}-\widetilde{f}(\widetilde{x}, \epsilon, \mu) \\
\widetilde{G}(\widetilde{x}, \epsilon, \mu)
\end{array}\right)
$$

for some analytic functions $\widetilde{f}$ and $\widetilde{G}$ such that $\widetilde{f}(0,0,0)=\widetilde{G}(0,0,0)=0$. Since $X_{0, \mu}$ has the curve of singularities $\{y=f(x, \mu)\}$, it follows from (7) that

$$
\psi_{2}^{0, \mu}(x, f(x, \mu))=\widetilde{f}\left(\psi_{1}^{0, \mu}(x, f(x, \mu)), 0, \mu\right)
$$

and

$$
\widetilde{G}\left(\psi_{1}^{0, \mu}(x, f(x, \mu)), 0, \mu\right)=0 .
$$

Since $f(x, 0)=O\left(x^{2}\right)$, the equation (8) implies

$$
\frac{\partial \psi_{2}^{0,0}}{\partial x}(0,0)=\frac{\partial \widetilde{f}}{\partial x}(0,0,0) \frac{\partial \psi_{1}^{0,0}}{\partial x}(0,0) .
$$

We obtain from (10) immediately $\frac{\partial \psi_{1}^{0,0}}{\partial x}(0,0) \neq 0$. Combining this with (9) we have

$$
\widetilde{G}(x, \epsilon, \mu)=\epsilon \widetilde{g}(x, \epsilon, \mu)
$$

with an analytic function $\widetilde{g}$. 
First we prove that $f(x, 0)$ and $\tilde{f}(x, 0,0)$ have the same order at $x=0$. The expression (7) implies

$$
\psi_{2}^{0,0}(x, 0)-\widetilde{f}\left(\psi_{1}^{0,0}(x, 0), 0,0\right)=-\rho\left(\psi^{0,0}(x, 0), 0,0\right) \frac{\partial \psi_{1}^{0,0}}{\partial x}(x, 0) f(x, 0) .
$$

Since $\psi^{0,0}$ preserves the line $\{y=0\}$, we obtain $\psi_{2}^{0,0}(x, 0) \equiv 0$. Combining this with $(11), \psi_{1}^{0,0}(0,0)=0, \frac{\partial \psi_{1}^{0,0}}{\partial x}(0,0) \neq 0$ and $\rho>0$, we get the same order of vanishing at $x=0$. Thus $\tilde{f}(x, 0,0)=O\left(x^{2}\right)$.

Next we show that the order of vanishing of $g(x, 0,0)$ at $x=0$ is equal to the order of vanishing of $\widetilde{g}(x, 0,0)$ at $x=0$. Clearly, as a simple consequence of (7),

$$
\widetilde{g}\left(\psi_{1}^{0,0}(x, f(x, 0)), 0,0\right)=\rho\left(\psi^{0,0}(x, f(x, 0)), 0,0\right) \frac{\partial \psi_{2}^{0,0}}{\partial y}(x, f(x, 0)) g(x, 0,0) .
$$

Since the order of $\tilde{f}(x, 0,0)$ at $x=0$ is at least 2 , we have $\frac{\partial \psi_{2}^{0,0}}{\partial x}(0,0)=0$ (see $(10))$. Hence, $\frac{\partial \psi_{2}^{0,0}}{\partial y}(0,0) \neq 0$.

On the other side, if we write $\rho_{1}(x)=\psi_{1}^{0,0}(x, f(x, 0))$, we obtain $\rho_{1}(0)=0$ and $\rho_{1}^{\prime}(0) \neq 0$. Here we used $\psi_{1}^{0,0}(0,0)=0, \frac{\partial \psi_{1}^{0,0}}{\partial x}(0,0) \neq 0$ and $f(x, 0)=O\left(x^{2}\right)$. Now the result easily follows.

\section{References}

[Bot57] Raoul Bott. Homogeneous vector bundles. Ann. of Math. (2), 66:203-248, 1957.

[DM14] Peter De Maesschalck. Gevrey normal forms for nilpotent contact points of order two. Discrete Contin. Dyn. Syst., 34(2):677-688, 2014.

[DMD11] P. De Maesschalck and F. Dumortier. Classical Liénard equations of degree $n \geq 6$ can have $\left[\frac{n-1}{2}\right]+2$ limit cycles. J. Differential Equations, 250(4):2162-2176, 2011.

[DMD16] Peter De Maesschalck and Thai Son Doan. Gevrey normal form for unfoldings of nilpotent contact points of planar slow-fast systems. Preprint, 2016.

[DMDR11] P. De Maesschalck, F. Dumortier, and R. Roussarie. Cyclicity of common slow-fast cycles. Indag. Math. (N.S.), 22(3-4):165-206, 2011.

[DMH14] P. De Maesschalck and R. Huzak. Slow divergence integrals in classical liénard equations near centers. J Dyn Diff Equat, DOI $=10.1007 / \mathrm{s} 10884-014-9358-1,2014$.

[DPR07] F. Dumortier, D. Panazzolo, and R. Roussarie. More limit cycles than expected in Liénard equations. Proc. Amer. Math. Soc., 135(6):1895-1904 (electronic), 2007. 
[DR96] Freddy Dumortier and Robert Roussarie. Canard cycles and center manifolds. Mem. Amer. Math. Soc., 121(577):x+100, 1996. With an appendix by Cheng Zhi Li.

[DR01] F. Dumortier and R. Roussarie. Multiple canard cycles in generalized Liénard equations. J. Differential Equations, 174(1):1-29, 2001.

[DR09] F. Dumortier and R. Roussarie. Birth of canard cycles. Discrete Contin. Dyn. Syst. Ser. S, 2(4):723-781, 2009.

[Dum] Freddy Dumortier. Techniques in the theory of local bifurcations: blow-up, normal forms, nilpotent bifurcations, singular perturbations. In Bifurcations and periodic orbits of vector fields (Montreal, PQ, 1992), volume 408 of NATO Adv. Sci. Inst. Ser. C Math. Phys. Sci., pages 19-73.

[Dum06] F. Dumortier. Compactification and desingularization of spaces of polynomial Liénard equations. J. Differential Equations, 224(2):296-313, 2006.

[FG65] Wolfgang Fischer and Hans Grauert. Lokal-triviale Familien kompakter komplexer Mannigfaltigkeiten. Nachr. Akad. Wiss. Göttingen Math.-Phys. Kl. II, 1965:89-94, 1965.

[FG02] Klaus Fritzsche and Hans Grauert. From holomorphic functions to complex manifolds, volume 213 of Graduate Texts in Mathematics. Springer-Verlag, New York, 2002.

[Gri65] Ph. A. Griffiths. The extension problem for compact submanifolds of complex manifolds. I. The case of a trivial normal bundle. In Proc. Conf. Complex Analysis (Minneapolis, 1964), pages 113-142. Springer, Berlin, 1965.

[HDM14] Renato Huzak and Peter De Maesschalck. Slow divergence integrals in generalized Liénard equations near centers. Electron. J. Qual. Theory Differ. Equ., (66), 2014.

[Kod86] Kunihiko Kodaira. Complex manifolds and deformation of complex structures, volume 283 of Grundlehren der Mathematischen Wissenschaften [Fundamental Principles of Mathematical Sciences]. Springer-Verlag, New York, 1986. Translated from the Japanese by Kazuo Akao, With an appendix by Daisuke Fujiwara.

[KS58] K. Kodaira and D. C. Spencer. On deformations of complex analytic structures. I, II. Ann. of Math. (2), 67:328-466, 1958.

[KS01] M. Krupa and P. Szmolyan. Relaxation oscillation and canard explosion. J. Differential Equations, 174(2):312-368, 2001.

[Lor06] Frank Loray. A preparation theorem for codimension-one foliations. Ann. of Math. (2), 163(2):709-722, 2006.

[Rou07] R. Roussarie. Putting a boundary to the space of Liénard equations. Discrete Contin. Dyn. Syst., 17(2):441-448, 2007. 
[Sav82] V. I. Savel'ev. Zero-type imbedding of a sphere into complex surfaces. Vestnik Moskov. Univ. Ser. I Mat. Mekh., (4):28-32, 85, 1982.

[Sma00] S. Smale. Mathematical problems for the next century. In Mathematics: frontiers and perspectives, pages 271-294. Amer. Math. Soc., Providence, RI, 2000.

[SŻa02] Ewa Stróżyna and Henryk Żolpolhk adek. The analytic and formal normal form for the nilpotent singularity. J. Differential Equations, 179(2):479-537, 2002.

[Tak74] Floris Takens. Forced oscillations and bifurcations. In Applications of global analysis, I (Sympos., Utrecht State Univ., Utrecht, 1973), pages 1-59. Comm. Math. Inst. Rijksuniv. Utrecht, No. 3-1974. Math. Inst. Rijksuniv. Utrecht, Utrecht, 1974. 